\title{
Verbos de movimiento virtual en griego antiguo
}

\author{
José Miguel Jiménez Delgado y Rafael Martínez Vázquez \\ Universidad de Sevilla \\ jmjimdelg@us.esy rmartinez@us.es
}

\section{Virtual motion verbs in Ancient Greek}

\begin{abstract}
El presente trabajo es un estudio de los verbos de movimiento empleados en las «construcciones de movimiento virtual» en griego antiguo. Los autores proponen que estas construcciones implican una proyección metafórica y estudian la incidencia de la metáfora en la construcción sintáctica y el empleo de las voces. Por otra parte, las referencias genérica y de estado asociadas a los verbos de movimiento virtual se explican a partir de su carácter descriptivo y el tipo textual descriptivo en que tienden a aparecer.
\end{abstract}

Palabras clave: virtualidad; movimiento virtual; verbos de movimiento; tipo textual descriptivo; referencia temporal-aspectual genérica; activa anticausativa.
This paper is a study of motion verbs employed in the so-called «virtual motion constructions» in Ancient Greek. The authors claim that these constructions involve a metaphorical projection and explore the role of metaphor in the syntactic construction and use of the voices. Besides, generic reference and stativity associated with virtual motion verbs are explained with reference to the descriptive character of these constructions and the descriptive text type in which they tend to appear.

Key words: virtuality; virtual motion; motion verbs; descriptive text type; generic temporal-aspectual reference; anticausative active.

\section{COnstrucciones De MOVimiento VirTUAL}

Las construcciones de movimiento virtual son expresiones con las que se designa un objeto inherentemente estático mediante la evocación de un movimiento abstracto, figurado o ficticio ${ }^{1}$. El movimiento virtual no tiene existen-

${ }^{1}$ Han sido denominadas de «movimiento abstracto» (Langacker 1986), de «movimiento subjetivo» (Matsumoto 1996) y de «movimiento ficticio» (Talmy 2000; Méndez Dosuna 2009). El primero en emplear el término «virtual» fue Talmy 1983. Sobre el sentido del término véase Langacker 1999. 
cia en el mundo real y se toma como referencia para describir una entidad real que constituye el referente indirecto de la construcción. Estas expresiones son frecuentes en distintas lenguas, como puede apreciarse en (1-4)

(1) Ou., Met. XI 230 est sinus Haemoniae curuos falcatus in arcus, | bracchia procurrunt (in mare) «El golfo de Hemonia tiene forma de hoz curvada; sus brazos se lanzan (al mar)».

(2) Fuero de Oreja, año 1139, é dende fasta dentro en las Alcarrias asi commo descende Tajuna en Jarama «Desde allí hasta la Alcarria según baja el Tajuña al Jarama».

(3) Highway 36 goes from Denver to Indianapolis «La autopista 36 va de Denver a Indianapolis».

(4) Die Straße wendet sich «La calle se gira».

La descripción de un objeto inerte en términos de un movimiento constituye una expresión compleja en la que el hablante evoca un desplazamiento y recorre mentalmente el trayecto evocado. El movimiento sólo tiene lugar como parte del proceso que siguen hablante y oyente al configurar un sentido para la expresión. Estudios psicológicos sobre hablantes reales parecen confirmar esta tesis (Matlock 2004), pero la idea en sí no es novedosa ni reciente. Ya a principios del siglo pasado Rodenbusch ${ }^{3}$ sugería una explicación semejante:

Indem wir sagen die Brücke schwingt sich über den Fluß, der Berg erhebt sich, verfolgen wir den Gegenstand mit unsern Augen in seiner ganzen Ausdehnung auf die im Verbum angedeutete Weise und rufen dabei in uns den Eindruck der Bewegung des Gegenstandes hervor. In einem Falle wie die Straße biegt sich, krümmt sich usw. mag dann die Vorstellung einer auf dem Wege vorschreitenden Person diesen Eindruck verstärken helfen.

Las construcciones de movimiento virtual han sido objeto de detallado estudio en el marco de la lingüística cognitiva en los últimos años ${ }^{4}$. En los

\footnotetext{
${ }^{2}$ Véanse, además, los trabajos de Matsumoto 1996 para el japonés, Sivonen 2005 para el finlandés o Takahashi 2001 para el tailandés.

${ }^{3}$ Rodenbusch 1911, p. 270.

${ }^{4}$ Aparte de los ya citados, véase Talmy 1983, 1996, 2000; Langacker 1986, 1990, 1999; Fauconnier 1997; Rojo y Valenzuela 2003; Matlock 2004.
} 
estudios sobre griego antiguo apenas hay aportaciones relevantes hasta fecha bastante reciente, sólo observaciones aisladas en estudios específicos ${ }^{5}$, gramáticas ${ }^{6}$ y algún que otro léxico ${ }^{7}$. En su estudio sobre el sentido de preposiciones y casos, Luraghi 2003, pp. 56-57, apela a los trabajos sobre el «movimiento ficticio» de Talmy y Langacker para explicar los usos locativos del acusativo de extensión que expresa la distancia entre dos referentes estáticos, así como algunos empleos de acusativo con preposición con función aparentemente locativa. Jiménez Delgado 2008a, p. 19, y 2008b, pp. 148-149, en sendos estudios sobre la diátesis y el marco predicativo del verbo griego, trata sobre el empleo metafórico de verbos agentivos para expresar un estado como la situación real descrita con el movimiento virtual. Martínez Vázquez 2008b, pp. 98-99, presenta varias explicaciones complementarias del empleo de $\varphi \varepsilon ́ p \omega$ en expresiones con sentido de movimiento virtual. Recientemente, Méndez Dosuna 2009 ofrece un interesante estudio en que examina «algunas de las características del movimiento fictivo en griego antiguo» (p. 6). Aunque el tratamiento que hace de los verbos de movimiento es parcial, enriquece estudios anteriores con abundancia de ejemplos tomados de inscripciones y aborda el tratamiento de expresiones que nadie hasta entonces había vinculado con el movimiento virtual.

En este trabajo presentamos una explicación de las construcciones con «verbo de movimiento virtual», que son las únicas en que hay un movimiento virtual explícitamente atribuido a una entidad estática. Para ello, ofrecemos en primer lugar una clasificación de las construcciones que de una forma $\mathrm{u}$ otra se han vinculado con el movimiento virtual, tipología que se torna imprescindible para delimitar el objeto de nuestro estudio. A continuación argumentamos que el sentido figurado de todos estos verbos se explica mejor a partir de una proyección de base metafórica que de un desplazamiento de base metonímica, como han sostenido algunos autores, y ofrecemos una breve descripción del papel de los verbos de movimiento en estas proyecciones. Finalmente, mostramos que en líneas generales el empleo de estos verbos está restringido a los usos gramaticales propios del tipo textual descriptivo en que estas expresiones aparecen.

\footnotetext{
${ }^{5}$ De Boel 1988, p. 95, habla de static direction.

${ }^{6}$ Schwyzer y Debrunner 1950, p. 459, hablan de Richtung in der Ruhelage.

7 ThGL, s. u. $\tau \varepsilon i ́ v \omega: 1926$ D; LSJ, s. u. $\tau \varepsilon i ́ v \omega:$ B.
} 
II. Tipología de LAS CONSTRUCCIONES DE MOVIMIENTO VIRTUAL

Las expresiones que han sido vinculadas con el movimiento virtual son de distinto tipo. Unas hacen referencia a un movimiento puramente imaginario y otras a un movimiento real, pero referido a un desplazado no específico. Se pueden distinguir tres tipos básicos que exponemos a continuación.

\section{Construcciones de desplazado virtual}

En estas construcciones se describe la ubicación de una entidad, típicamente un accidente geográfico, evocando el desplazamiento de una persona no específica en una dirección determinada. Hay varias expresiones diferentes ${ }^{8}$, pero en todas ellas la ubicación del accidente en cuestión se describe en términos literales, es decir, mediante verbos que no expresan movimiento, sino reposo. Dos tipos bien conocidos en la tradición gramatical griega son el empleo del genitivo del punto de vista y el dativo «corográfico» con participio:

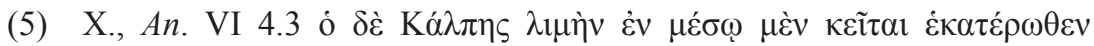

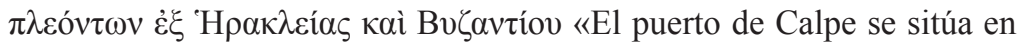
medio de los que navegan desde Heraclea y desde Bizancio».

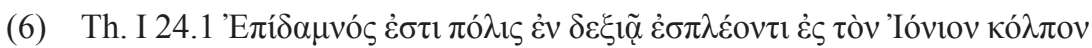
«Epidamno es una ciudad a la derecha para el que navega hacia el interior del golfo Jónico».

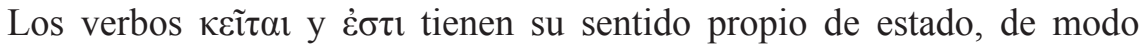
que su empleo carece de interés para un estudio sobre verbos que expresan movimiento virtual ${ }^{9}$.

${ }^{8}$ Méndez Dosuna 2009 menciona varios tipos de carácter sintáctico: dativo de punto de vista, genitivo de punto de vista, sujeto en segunda persona genérica, objeto en segunda persona genérica y objeto indirecto.

9 Méndez Dosuna 2009, p. 10, reconoce que «el viajero es puramente virtual, pero el movimiento es verídico». 


\section{Construcciones con verbo de estado y un adjunto que implica un movimiento virtual}

Otro tipo, del que hay ejemplos abundantes en griego antiguo, se da cuando se combinan expresiones preposicionales de dirección con verbos de referencia estativa ${ }^{10}$ :

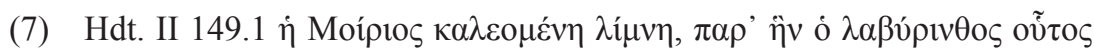

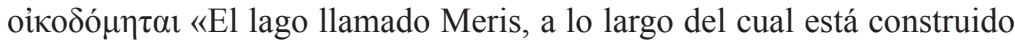
este laberinto».

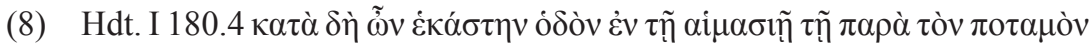
$\pi v \lambda i ́ \delta \varepsilon \varsigma \dot{\varepsilon} \pi \tilde{\eta} \sigma \alpha v$ «Así pues, al final de cada camino, en el vallado situado a lo largo del río, había portillos».

Luraghi 2003 explica estos usos como casos de movimiento virtual, retomando las tesis de Talmy y Langacker sobre ejemplos semejantes en inglés. Ahora bien, aunque el movimiento implícito en el giro preposicional es puramente virtual, el sentido del verbo sigue siendo literal, ya que no es un verbo de movimiento, $y$, por tanto, también carece de interés para este estudio ${ }^{11}$.

\section{Construcciones de movimiento virtual en sentido propio: el «verbo de movimiento virtual»}

En las construcciones de movimiento virtual en sentido propio se describe la posición de un objeto evocando un desplazamiento atribuido explícitamente al propio objeto, que se construye como sujeto de un verbo de movimiento. Podemos identificar cuatro tipos de acuerdo con las propiedades del objeto que se pretende describir mediante la referencia al movimiento virtual.

${ }^{10}$ Horrocks 1983, pp. 183-185, explica que estos giros implican la inserción de una expresión de dirección en una expresión de localización o extensión.

${ }^{11}$ Méndez Dosuna 2009, pp. 27-28, coincide con Luraghi y precisa que «el movimiento no está en las escenas descritas, sino en el "espectador", que desplaza mentalmente su mirada por el escenario como si se tratase de un travelling cinematográfico». Es el «barrido mental» (mental scanning) que aduce Langacker 1991, p. 82. Luraghi 2003, p. 56, entiende que el proceso es metafórico: «the notion of motion along a trajectory is metaphorically shifted to state. This is an instance of 'fictive motion', as defined in Talmy (2000, Chapter 2): motion is metaphorically used to refer to static location on an extended area». 
El primer tipo describe la posición del objeto:

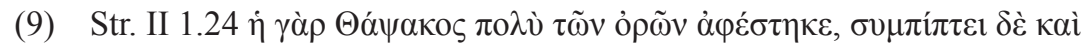

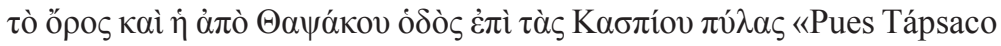
está muy apartada de las montañas, pero la cordillera y el camino (que viene) de Tápsaco se encuentran en las Puertas del Caspio».

Con frecuencia se expresa la posición relativa de dos objetos, accidentes naturales en este caso, evocando el movimiento imaginario de uno de ellos con relación al otro. Obsérvese que en todas estas construcciones el sentido del verbo de movimiento no es literal en ningún caso.

El segundo tipo expresa la orientación del objeto o una de sus partes:

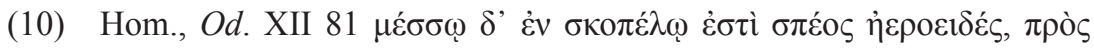

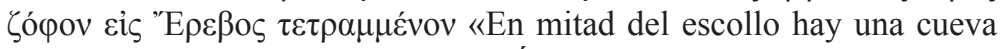
nebulosa, girada a poniente hacia el Érebo».

El tercer tipo es el esquema conceptual más representativo y mejor estudiado entre las construcciones de movimiento virtual, identificado por Talmy 2000 , p. 138, como «trayectos de coextensión» (coextension paths), que describen la forma, orientación o ubicación de una entidad en términos del trayecto recorrido a lo largo de la extensión que ocupa en el espacio:

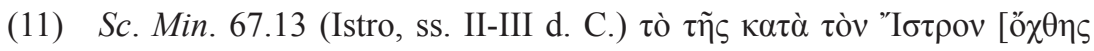

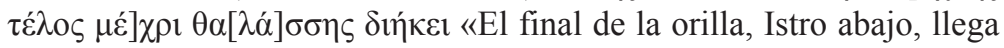
hasta el mar».

Finalmente, existe un tipo mixto con referencia a un viajero genérico y a un movimiento virtual atribuido al objeto localizado ${ }^{12}$. En este tipo se invierten los papeles del observador en movimiento y el objeto localizado, de modo que el objeto es presentado en movimiento saliendo al paso ante un observador hipotético:

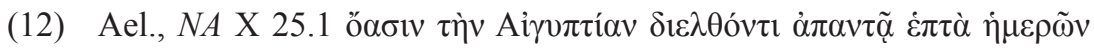

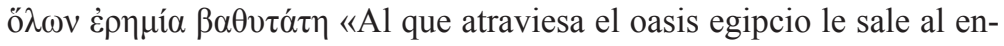
cuentro un profundo yermo de siete días completos».

12 Talmy 2000, p. 130, lo llama «movimiento con referencia al marco» (frame relative motion). 


\section{El SENTIDO FIGURAdO DE LOS VERBOS DE MOVIMIENTO VIRTUAL}

En las construcciones de movimiento virtual propiamente dichas el sentido del verbo es un sentido figurado, precisamente porque el sujeto de la construcción refiere un ente inanimado e inerte (Rodenbusch 1911, p. 273). En la medida en que se adscribe un movimiento a una entidad inerte todas estas construcciones tienen una base metafórica ${ }^{13}$. En general, el sentido de las expresiones metafóricas se construye mediante el choque de dos estructuras semánticas, la literal y la figurada, y, como toda metáfora conceptual, el movimiento virtual implica la «proyección» (mapping) ${ }^{14}$ de una estructura conceptual sobre otra. Con todo, no todas estas expresiones son idénticas, sino que se pueden distinguir dos casos distintos que examinamos a continuación.

En primer lugar, el sujeto puede referir una entidad que está asociada con el movimiento evocado. Es el caso del camino que «va» o «conduce» de un lado a otro:

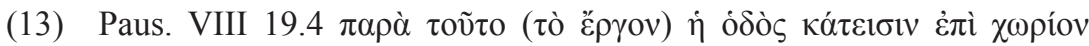
$\Lambda$ vкоvрíav «Junto a esta (obra) el camino baja al territorio de Licuria».

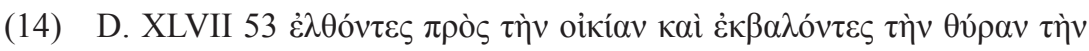

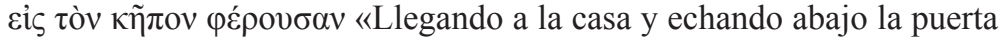
que lleva al jardín».

La metáfora pivota sobre una relación real que existe entre el camino y la acción de recorrerlo. Estos casos son los que han recibido una mayor atención. Martínez Vázquez 2008b, pp. 98-99, da una explicación del empleo virtual de $\varphi \varepsilon ́ p \omega$ en (14) partiendo de la concepción del camino como un guía metafórico. Pero se trata de una metáfora con proyecciones dentro del mismo

${ }^{13}$ Langacker 1999, p. 82, entiende que las construcciones de movimiento virtual se pueden explicar de formas distintas, pero compatibles entre sí, y admite la metáfora como una de ellas. Otros autores, como Coulson y Oakley 2005, p. 1531, se muestran reticentes a entenderlo como metáfora: «While one might be hesitant to classify fictive motion as metaphor ... most native speakers would presumably agree that such examples are non-literal».

${ }^{14}$ En el sentido matemático del término, esto es, cualquier forma sistemática de asignar a cada objeto de un conjunto otro objeto de otro o del mismo conjunto (Martínez Vázquez 2008a, p. 14, n. 9). 
dominio conceptual, ya que el objeto descrito por la expresión está asociado a un movimiento. Sobre la base de esta relación propone que, alternativamente, podría pensarse en una metonimia que promueve el prosecutivo a la posición del sujeto agente, ya que en su descripción interesa focalizar el camino y no el guía eventual. Méndez Dosuna (2009, pp. 8-9) propone asimismo una metonimia, aunque aduce un desplazamiento diferente: «son entidades estáticas, preferentemente de configuración longitudinal, y corresponden a un Trayecto que puede recorrer una persona. Por metonimia, el movimiento de esa persona se transfiere a la entidad inmóvil ${ }^{15} \gg$.

Una relación metafórica entre términos contiguos evoca la figura de la metonimia con facilidad, ya que esta figura se basa en relaciones de contigüidad nocional. Pero hay que advertir que no se trataría, en ningún caso, de una metonimia común. El caso común en que se transfiere metonímicamente el movimiento al camino es el que se observa en expresiones como «el camino es de piedra» (literal) / «el camino es muy duro» (metonímico). El sentido de estas construcciones de movimiento virtual es más complejo, porque implican una concepción metafórica del sujeto como ser que se desplaza.

En segundo lugar, las expresiones de movimiento virtual se emplean también para describir accidentes geográficos y partes del cuerpo, entre otros muchos objetos, que a priori no tienen ninguna relación con el movimiento evocado por el verbo:

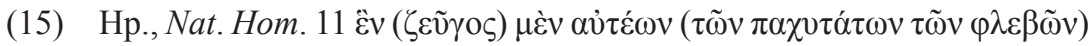

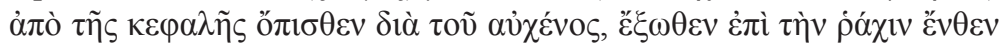

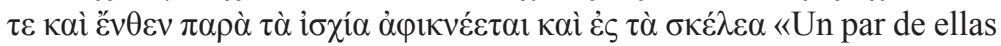
(las venas más gruesas) llega desde detrás de la cabeza a través del cuello, por fuera por encima de ambos lados de la columna vertebral, junto a las caderas y hasta las extremidades».

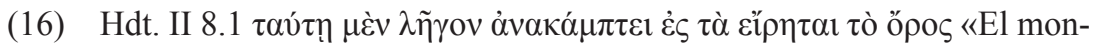
te, terminando allí, dobla hacia las zonas mencionadas».

En estas expresiones el movimiento metafórico por parte del sujeto resulta más evidente, pues para que se dé un desplazamiento semántico de base

15 Ya Matlock 2004 había explicado la conceptualización del sentido de estas expresiones como una metonimia que transfiere el movimiento a lo largo de un trayecto al trayecto mismo (movement along a path for the path). 
metonímica es necesario que haya una relación previa entre los términos que no existe en el caso de las venas ni de los montes ${ }^{16}$.

\section{El VERBo DE MOVIMIENTO EN LA CONSTRUCCIÓN DE MOVIMIENTO VIRTUAL}

El papel de los verbos de movimiento en la metáfora del movimiento virtual presenta algunas peculiaridades respecto a la relación con sus participantes, tal y como describimos brevemente a continuación.

Es frecuente que los verbos de movimiento sean intransitivos, pues tienen un único actante que designa la entidad que se desplaza. Si el sentido de las expresiones figuradas se construye sobre la base de la proyección de dos estructuras semánticas, cuando se usa un verbo de movimiento intransitivo ambas estructuras presentan un solo participante o actante principal, de modo que no hay restricciones y su empleo es abundante, tanto en voz activa ${ }^{17}$ como en voz media ${ }^{18}$ :

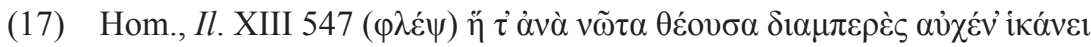
«La (vena) que corriendo espalda arriba de un extremo a otro alcanza el cuello».

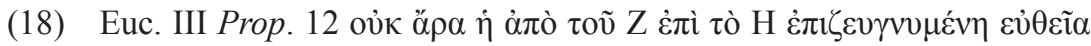

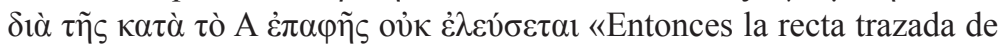
F a G no pasará por el punto de contacto en A».

\footnotetext{
${ }^{16}$ Matlock 2004, p. 239, rechaza la explicación metonímica de estas expresiones: «because no motion is expected to occur ... it follows that properties associated with motion cannot metonymically map to properties about the shape or configuration of the path». Méndez Dosuna 2009, p. 21, agrupa los dos tipos y los explica ambos como metonímicos. Para este segundo tipo admite que «un humano no puede desplazarse por una abrazadera o una vena» y explica que «aquí la 'recorribilidad' tiene una base visual o mental».

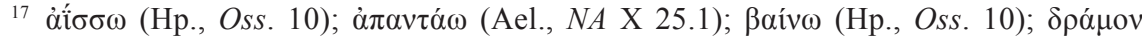

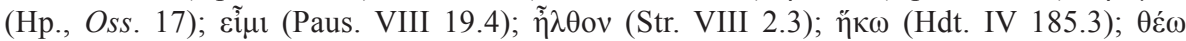

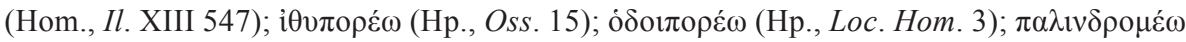

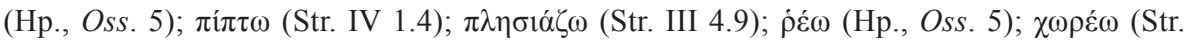
$\mathrm{V}$ 1.3).

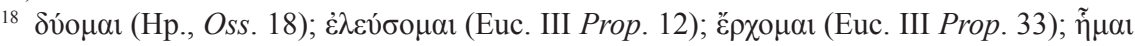

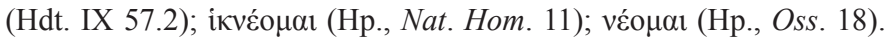


Menos frecuentes son los verbos de movimiento transitivos con un acusativo que designa el camino recorrido. Un ejemplo de movimiento virtual con este tipo de verbos es el siguiente:

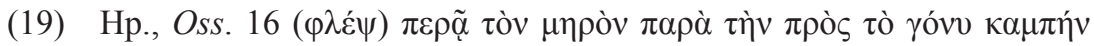
«(Una vena) atraviesa el muslo junto a la articulación de la rodilla».

La estructura semántica de estos verbos tampoco plantea ningún problema en su empleo como expresiones de movimiento virtual. Se identifica el sujeto del movimiento con la entidad inerte cuya localización se pretende describir; el objeto, por su parte, describe una parte de la ruta, que se entenderá como un referente de la localización del sujeto inerte en cuestión ${ }^{19}$.

La mayor parte de los verbos de movimiento transitivos son verbos de movimiento inducido, en el sentido de que la entidad que se mueve se expresa en la construcción activa mediante el objeto, no mediante el sujeto, que es inductor del movimiento. Estos verbos también se emplean con frecuencia en construcciones de movimiento virtual, pero su empleo es algo más complejo y está sujeto a restricciones, ya que el inductor del movimiento no tiene ninguna utilidad para la metáfora del movimiento virtual. De modo que, para acomodar su estructura a la del referente indirecto de la construcción, se hace abstracción de éste escogiendo la construcción intransitiva del verbo, en la que el sujeto designa la entidad inducida a movimiento. Esta construcción es muy natural en el sistema del verbo griego antiguo con el verbo en voz media, una media intransitiva opuesta a una activa transitiva causativa ${ }^{20}$. Hallamos ejemplos abundantes de ella ${ }^{21}$ :

${ }_{19}$ El acusativo refiere la ruta del desplazamiento expresado por el verbo y, en este sentido, sólo de una manera tangencial se puede considerar afectado, por lo que se trata de verbos de transitividad baja (Martínez Vázquez 1998, p. 27).

${ }^{20}$ Allan 2003, p. 76, distingue en su clasificación semántica de la voz media en griego antiguo la «media de movimiento corporal» (body motion middle), que puede construirse con sujeto agentivo o no-agentivo. En el segundo caso, el significado está relacionado con la «media de proceso espontáneo» (spontaneous process middle).

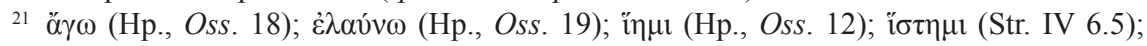

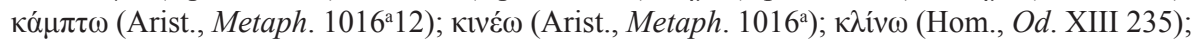

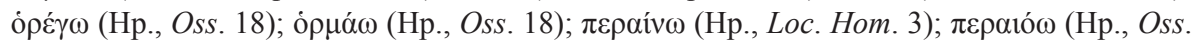

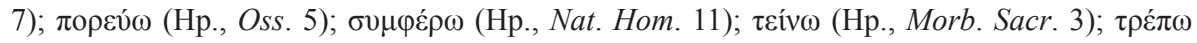




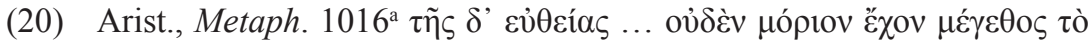

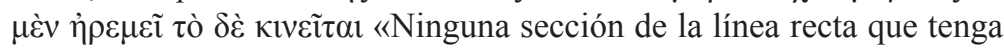
magnitud está quieta mientras que otra se mueve».

Curiosamente, junto al empleo de la voz media es posible en griego el empleo de una activa intransitiva anticausativa (por oposición a la activa de movimiento inducido). Puede decirse que la referencia estativa de estas expresiones, que describen entidades cuyo papel es indiferente a nociones como la actividad y la pasividad, favorece la neutralización de las voces y el empleo de la activa con diátesis secundarias (Jiménez Delgado 2008a). Este hecho explica la relativa frecuencia con la que se emplea la activa anticausativa en las construcciones de movimiento virtual en griego antiguo ${ }^{22}$. De hecho, uno de los verbos que expresa movimiento virtual con más frecuencia es $\tau$ cív $\omega$, que puede aparecer en voz media y tema de perfecto ${ }^{23}$, pero que se emplea mayoritariamente en activa intransitiva ${ }^{24}$ :

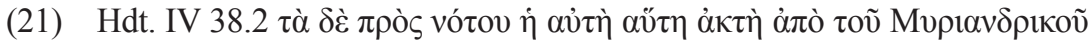

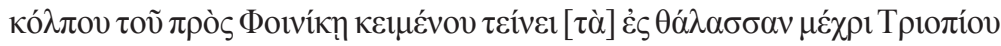

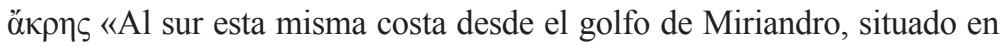
dirección a Fenicia, se extiende al mar hasta el cabo de Triopio».

(22) Hp., Oss. 17 ả

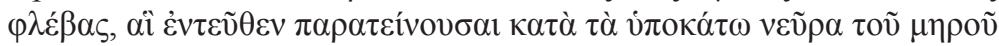

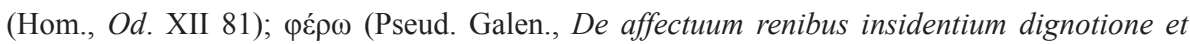
curatione lib. XIX 656).

${ }^{22}$ La gramática griega no ha prestado apenas atención a la activa anticausativa, dado que no es frecuente. Kühner y Gerth 1898, p. 91 ss., la incluyen dentro de los casos de intransitivización de los verbos transitivos. Méndez Dosuna 2009 la estudia en el caso de los impe-

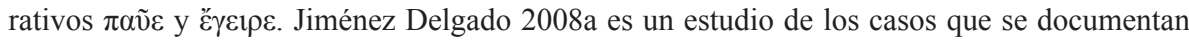
en Heródoto. Fuera de un mismo paradigma tenemos la denominada tradicionalmente "pasiva

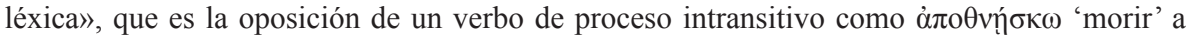

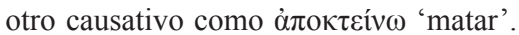

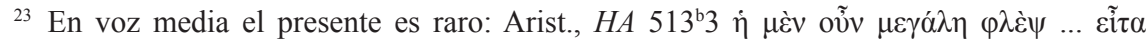

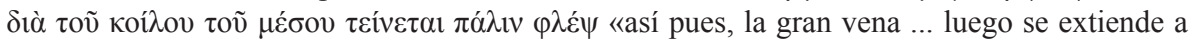
través del ventrículo medio de nuevo como vena».

${ }^{24}$ Otros verbos transitivos empleados en activa intransitiva son los siguientes: кó $\mu \pi \tau \omega$

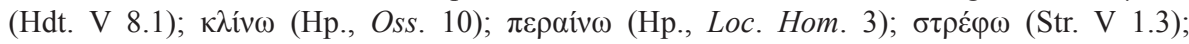

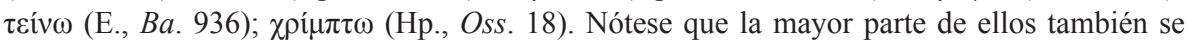
construyen en media intransitiva, en concreto, todos salvo $\sigma \tau \rho \varepsilon ́ \varphi \omega$ у $\chi \rho i ́ \mu \pi \tau \omega$. 


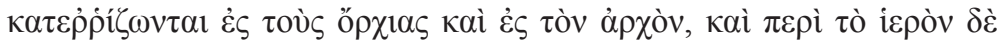

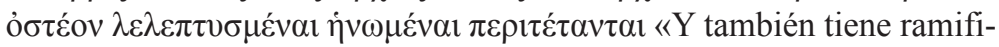
cadas hacia la corva venas muy entrelazadas, que desde allí, extendiéndose del lado de los nervios de debajo del muslo, están bien enraizadas en los testículos y en el ano, y adelgazadas y unidas están extendidas alrededor del hueso sacro».

Un último caso se da con los verbos ö $\gamma \omega$ y $\varphi \varepsilon ́ p \omega$, que se usan con mucha frecuencia en voz activa intransitiva para describir la extensión, orientación o localización de un objeto, esté o no esté asociado a un movimiento:

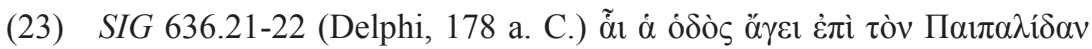

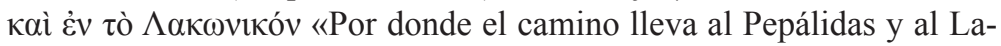
cónico».

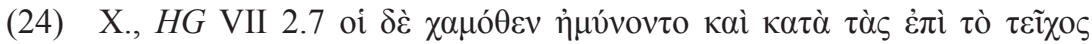

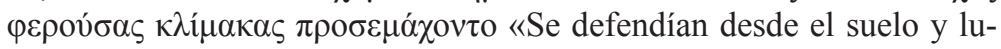
chaban desde las escaleras que llevaban al muro».

Se entiende que esta construcción es intransitiva por elisión de un objeto que designaría el caminante, que puede expresarse con una segunda persona impersonal en otras lenguas: this road takes you to the city «esta carretera te lleva a la ciudad», Priap. 86.21 semita haec deinde vos feret ipsa «que esta senda luego os lleve ella misma». La construcción adquiere entonces un sentido semejante a la de cualquier verbo transitivo que se construye de forma absoluta por elipsis de un objeto genérico. Esta construcción tiende a dotar al verbo de sentido estativo ${ }^{25}$ y lo hace más apto para la expresión del movimiento virtual, que en realidad expresa estado.

\footnotetext{
${ }^{25}$ Como observan Kühner y Gerth 1898, p. 91: «[Aber an sich haben auch diese Verben,] wenn sie objektlos stehen, intransitive Bedeutung, als: $\beta \alpha \dot{\alpha} \lambda \lambda \omega$, ich werfe, $\gamma \rho \alpha \dot{\varphi} \varphi \omega$, ich schreibe, und bezeichnen alsdann die Thätigkeitsäusserung als einen Zustand, in dem sich das Subjekt befindet».
} 


\section{LOS «TIEMPOS» DEL VERBO DE MOVIMIENTO VIRTUAL \\ Y EL TIPO TEXTUAL DESCRIPTIVO}

Un hecho no observado en los estudios sobre el movimiento virtual es que las metáforas de movimiento virtual son metáforas fundamentalmente descriptivas. Su faceta más relevante no es que designan entidades estáticas, sino que se emplean para describir estas entidades. Esta función descriptiva explica que el sentido denotativo de la expresión centre el interés del hablante, a diferencia de las metáforas poéticas y creativas que tienen una carga connotativa más fuerte ${ }^{26}$.

En consonancia con su función descriptiva, las expresiones de movimiento virtual se emplean en pasajes enmarcados dentro del tipo textual descriptivo. Son usadas para la descripción de accidentes geográficos en la obra de geógrafos e historiadores, en la descripción de la anatomía de seres humanos y animales, sobre todo la configuración de las venas, dentro de la obra de médicos como Hipócrates o Galeno y finalmente aparecen en los tratados de geometría de Euclides. Su adscripción al tipo textual descriptivo explica las restricciones que reflejan estos verbos tanto en cuanto al empleo de sus formas como en cuanto a la interpretación de las mismas ${ }^{27}$. Entre otros rasgos, la descripción se caracteriza por el uso recurrente de formas verbales de referencia estativa, habitual y genérica ${ }^{28}$. Como es natural, no se usan los verbos de movimiento virtual en tiempos y modos que no sean propios de la descripción en griego antiguo. Los repasamos a continuación.

${ }^{26}$ Méndez Dosuna 2009, pp. 6-7, comenta con acierto que las construcciones de movimiento virtual «aparecen en historiadores, en geógrafos y en otros muchos autores, pero también en inscripciones que carecen de pretensiones literarias. No era, pues, una figura literaria, sino — como sucede entre nosotros - un recurso usado en la lengua corriente».

${ }^{27} \mathrm{La}$ tesis de que el tipo textual restringe el uso y explica los valores de unidades lingüísticas está formulada en Martínez Vázquez 2011 y se ejemplifica con el empleo de oü $\omega \varsigma$ como adverbio conjuntivo en Tucídides.

${ }^{28}$ Cf. Kroon 2007, p. 71. Langacker 1991, pp. 263-266, explica que todos estos usos invocan un aspecto de la experiencia humana que denomina el «modelo del mundo estructurado» (structured world model), es decir, invocan el conocimiento sobre cómo es el mundo y no el conocimiento sobre lo que en él sucede, de modo que «habitual, generic and timeless statements can all be seen as special cases of the same phenomenon». 


\section{Presente general}

Característico de las construcciones de movimiento virtual es el empleo del tema de presente, la forma habitual para expresar estados permanentes, es decir, propiedades constantes e inmutables en el tiempo. Los verbos de movimiento virtual alternan con el empleo de verbos estativos, lo que confirma esta apreciación:

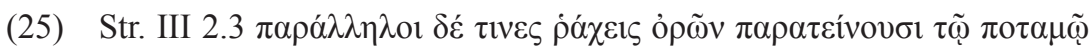

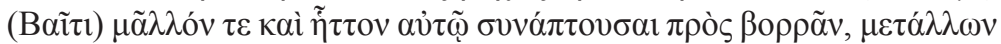

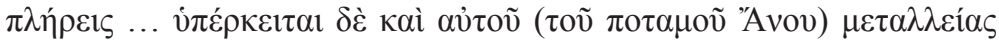

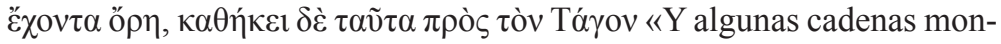
tañosas se extienden hacia el norte paralelas al río (Betis) tocándolo más o menos, llenas de minas ... Y por encima de éste (el río Anas) también hay montañas que tienen minas y éstas bajan hacia el Tago».

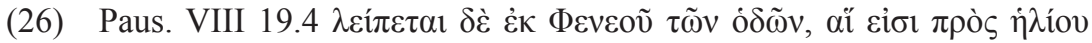

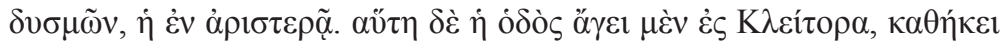

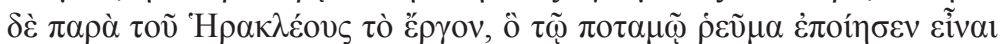

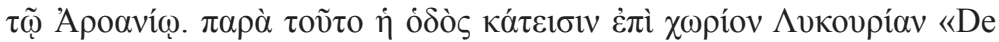
los caminos (que parten) desde Feneo que están hacia poniente queda el de la izquierda. Este camino lleva a Clítor y baja a lo largo de la obra de Heracles, que hizo que fuera el cauce del río Aroanio. A lo largo de ésta el camino baja al territorio de Licuria».

En lenguas como el inglés o el español, la forma verbal empleada en construcciones equivalentes es el presente simple (goes), característico de los verbos de estado, y no el progresivo (is going), que sería propio de un verbo con referencia dinámica y perfectiva. Hay teorías diferentes sobre el valor exacto de este presente, pero la mayoría de ellas parten de su valor aspectual de estado ${ }^{29}$.

29 Jackendoff 1983 e Iwata 1996 entienden que son variantes léxicas de una misma base verbal. Langacker 1999 y Talmy 2000 simplemente explican que es la situación real y no el movimiento virtual la que configura el carácter aspectual de la expresión y determina la elección de la forma del verbo. En realidad, el presente simple de estas lenguas se usa con verbos de estado, pero también con verbos «dinámicos» de sentido habitual o de referencia genérica. Todos ellos son manifestaciones del mismo fenómeno, según la tesis de Langacker citada en la nota precedente. 
En los tratados de anatomía puede pensarse que la referencia es asimismo genérica, en la medida en que se describen propiedades referidas no a un órgano o a un cuerpo concreto, sino a todos los miembros de su categoría:

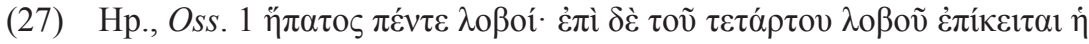

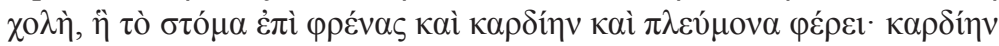

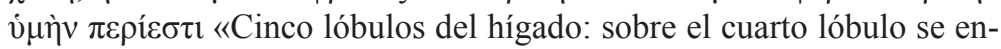
cuentra la vejiga de la bilis, que lleva su abertura al diafragma, el corazón y el pulmón. Hay una membrana alrededor del corazón».

En cuanto a la temporalidad de estas formas cabe decir que es la propia de las formas estativas y genéricas, descrita por las gramáticas como presente genera ${ }^{30}$, no como presente actual. Prueba de ello es que en este tipo de descripción se emplean con frecuencia oraciones nominales puras ${ }^{31}$ :

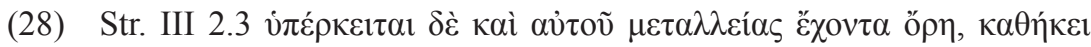

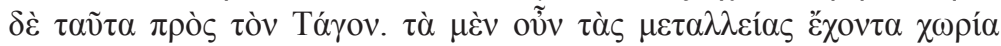

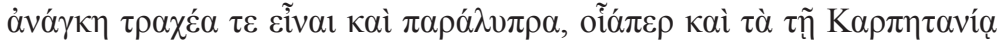

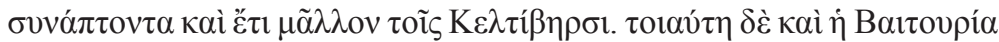

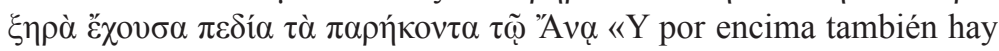
montañas que tienen minas y éstas bajan hacia el Tago. En efecto, los territorios que tienen minas es necesario que sean quebrados y poco fértiles, como los que lindan con la Carpetania y, sobre todo, los que lindan con los celtíberos. De esta manera es también la Beturia, que tiene llanuras secas que van paralelas al Anas».

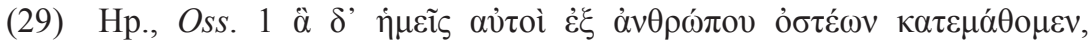

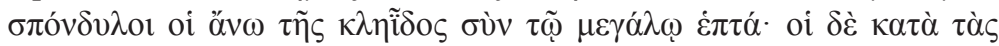

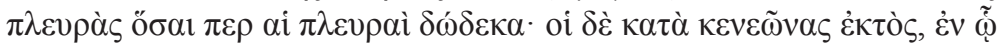

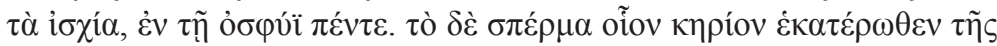

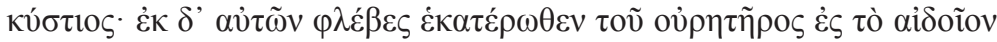
$\tau \varepsilon i ́ v 0 v \sigma 1$ «Y lo que nosotros mismos hemos aprendido de la observación de los huesos humanos, que las vértebras de encima de la clavícula con la grande son siete, las que están a la altura de las costillas, conforme

${ }^{30}$ Kühner y Gerth 1898, pp. 132-133; Smyth 1920, §1877; Crespo, Conti y Maquieira 2003, pp. 256-257.

${ }^{31}$ Que aparecen «dans le discours où le sujet parlant pose des rapports qui ne sont ni situés dans le temps ni modalisés» (Chantraine 1963, p. 1). 
al número de costillas, doce, las que están a la altura de las ijadas por fuera, donde las caderas, en la zona lumbar, cinco. El esperma es como cera de ambos lados de la vejiga y las venas (que parten) desde ellos se extienden de ambos lados de la uretra a los genitales».

Este presente es propio de situaciones que se dan en cualquier momento incluido el de la enunciación. En la descripción de objetos concretos se percibe un valor temporal de presente. En la formulación de axiomas científicos el sentido es atemporal.

\section{Perfecto general}

En las construcciones de movimiento virtual es frecuente el uso del tema de perfecto expresando estado resultante de un movimiento imaginario:

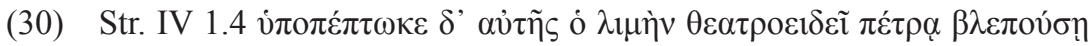
$\pi \rho o ̀ \varsigma$ vótov «Y su puerto está echado a los pies de una roca en forma de graderío que mira al sur».

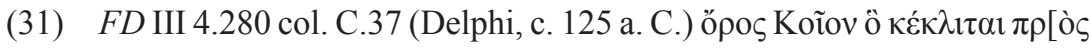
$\Pi \alpha \rho] v \alpha \sigma \sigma o ́ v$ «El monte Ceo que está inclinado hacia el Parnaso».

El tema de perfecto es muy natural para expresar una situación estativa, pero hemos observado en la sección anterior que algunos verbos documentan indistintamente el empleo del presente y del perfecto. El verbo $\tau$ cív $\omega$ es uno de los más frecuentes en griego para expresar movimiento virtual y se emplea tanto en tema de perfecto y voz media como en tema de presente y voz activa. La proximidad de sentido entre los dos temas se aprecia en (32-33):

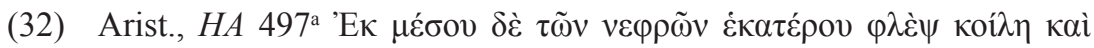

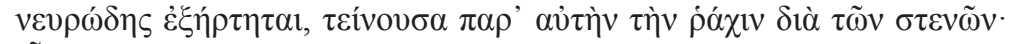

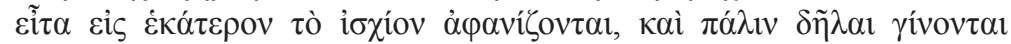

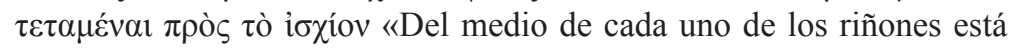
suspendida una vena cava y fibrosa que se extiende a lo largo de la propia columna vertebral a través de los pasos; luego desaparecen en cada una de las ancas y de nuevo se hacen visibles extendidas junto a la cadera». 


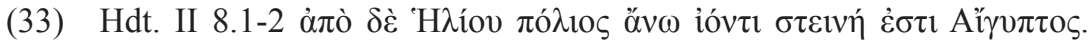

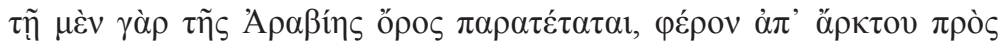

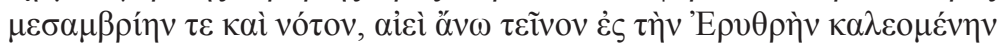

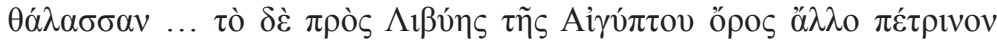

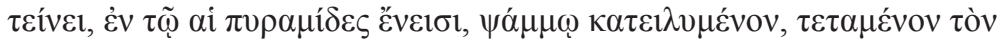

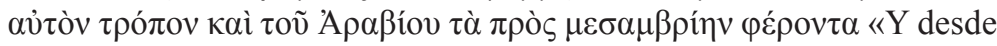
Heliópolis, para el que va hacia arriba, Egipto resulta estrecho, pues allí está extendida a lo largo la cadena montañosa de Arabia, que lleva desde el norte al mediodía y el sur, siempre extendiéndose hacia arriba al mar llamado Rojo ... Y en la parte de Egipto que linda con Libia se extiende otra cadena rocosa, en la cual hay pirámides, cubierta de arena, extendida de la misma forma que las montañas de Arabia que llevan al mediodía».

El presente con valor próximo al de perfecto se usa en griego con cierta frecuencia ${ }^{32}$. Como afirma Adrados 1992, p. 412, precisamente a propósito del empleo de presente por perfecto en griego, «un estado implica siempre una acción pasada: si uno yace, está echado, es que se ha echado previamente». Ya Rodenbusch 1911, p. 270, explicaba las expresiones de movimiento virtual como presentes por perfectos con valor resultativo ${ }^{33}$. No es una tesis que explique todos los casos tratados aquí, pero sí un testimonio de que en algunos contextos presente y perfecto del verbo de movimiento virtual se distinguen sólo por matices ligeros.

También se emplea el tema de perfecto en los textos descriptivos con un valor descrito como perfecto general por las gramáticas ${ }^{34}$. Como en el caso del presente, puede tener referencia genérica cuando va referido a órganos del cuerpo, ya que lo dicho es aplicable a todos los individuos de una especie:

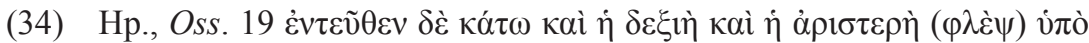

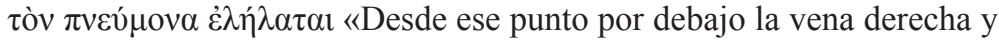
la izquierda están conducidas bajo el pulmón».

32 Cf. Martínez Vázquez 2009.

${ }^{33}$ «Erst wenn wir die Vorstellung der Bewegung zu Ende geführt haben, tritt der Eindruck des bewegungslosen Zustandes endgültig hervor, auch das Verbum hat damit resultative Bedeutung bekommen».

${ }^{34}$ Crespo, Conti y Maquieira 2003, pp. 262-263; Stahl 1907, p. 118 ss., lo llama «perfecto empírico», al igual que Smyth 1920, § 1948. 


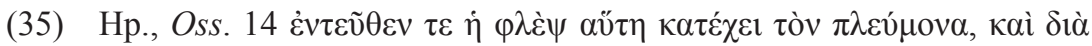

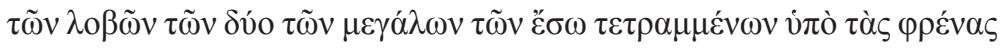

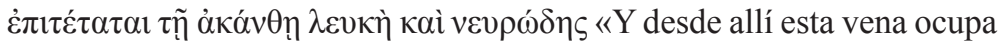
el pulmón y, a través de los dos lóbulos grandes que están vueltos hacia dentro, está extendida blanca y provista de nervios bajo el diafragma hacia la espina dorsal».

\section{Imperfecto descriptivo}

Las expresiones de movimiento virtual pueden describir entidades ubicadas en el pasado o en un tiempo mítico. En tales casos se emplean las formas de imperfecto que las gramáticas suelen tipificar como descripti$\operatorname{vos}^{35}$. Méndez Dosuna 2009, p. 12, ofrece un ejemplo de la descripción de la Atlántida:

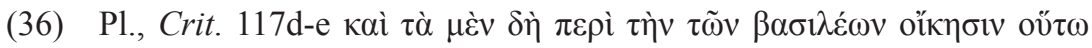

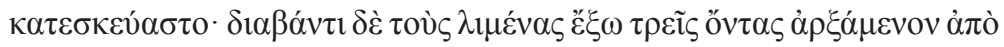

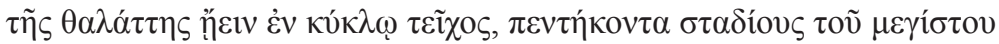

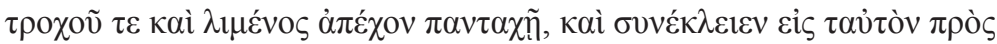

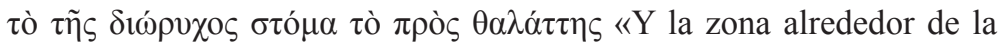
morada de los reyes estaba dispuesta de la siguiente manera: para el que atravesaba los tres puertos exteriores iba un muro en círculo que empezaba desde el mar, separado cincuenta estadios del círculo mayor $\mathrm{y}$ del puerto en todos sus puntos, y se cerraba en el mismo punto junto a la embocadura del canal en el mar».

Con frecuencia el morfema de pasado no expresa tanto la datación del estado como el punto de vista que el hablante adopta respecto a su descripción, y se usa este imperfecto para describir entidades que siguen existiendo en el tiempo del autor. Un ejemplo clásico es ${ }^{36}$ :

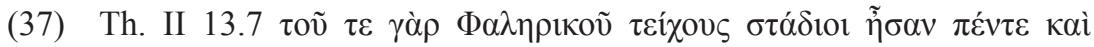

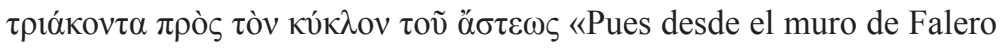
había treinta y cinco estadios hasta el recinto de la ciudad».

35 Kühner y Gerth 1898, pp. 143 y 152; Smyth 1920, §1898.

${ }^{36}$ Crespo, Conti y Maquieira 2003, p. 259. 
No hemos hallado ejemplos de imperfecto en la descripción de objetos que subsistan en el tiempo del hablante con referencia a movimiento virtual, pero no hay motivo para descartarlos. Por su parte, el carácter atemporal de los axiomas científicos hace inviable el empleo del imperfecto.

\section{Aoristos «gnómicos»}

En los tratados de anatomía, junto a presentes y perfectos, se emplea también el tema de aoristo. Es un aoristo muy próximo al uso etiquetado como «gnómico» en los manuales ${ }^{37}$. Dicho valor genérico es perfectamente armónico con la construcción de movimiento virtual, en la que alterna con el presente y el perfecto de referencia igualmente genérica. Así se aprecia en el siguiente pasaje hipocrático, en que a una secuencia de tres perfectos siguen cinco aoristos y un presente:

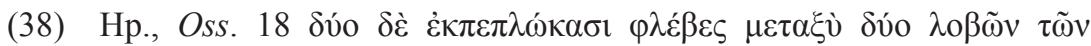

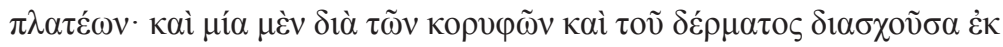

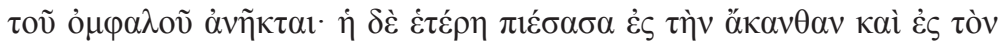

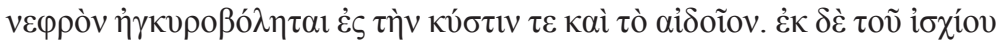

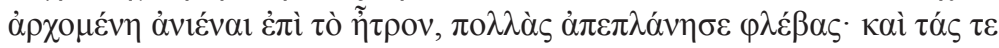

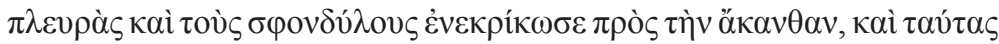

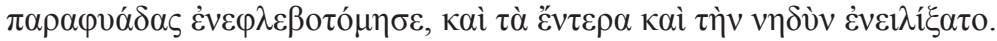

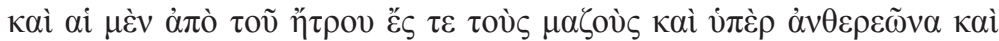

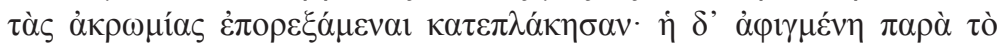

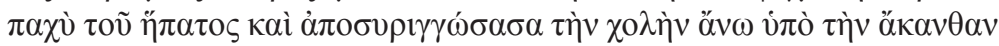

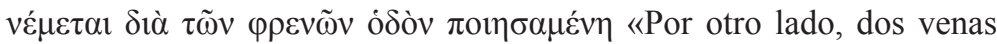
están desplegadas entre los dos lóbulos planos. Una de ellas atravesando por la parte alta y por la piel está descubierta por el ombligo, la otra tras presionar en la espina dorsal y el riñón está anclada en la vejiga y los genitales. Empezando a subir desde la cadera hacia el abdomen distribuye muchas venas y rodea las costillas y las vértebras en dirección a la espina dorsal, introduce estas ramificaciones y envuelve los intestinos y el vientre. $Y$ las que se elevan desde el abdomen a las mamas por encima del cuello y de la parte superior de los homóplatos se entrecruzan. Por otra parte, la que llega junto a la parte ancha del hígado y hace de con-

${ }^{37}$ Kühner y Gerth 1898, pp. 158-159; Stahl 1907, pp. 131 ss; Smyth 1920, § 1931; Crespo, Conti y Maquieira 2003, p. 261. 
ducto a la bilis ocupa la parte de arriba bajo la espina dorsal, tras realizar su trayecto por el diafragma».

El empleo de aoristos dota a la descripción de viveza y ritmo, evocando una secuencialidad y, en suma, una temporalidad tan metafórica como el propio movimiento atribuido a las venas. Las gramáticas refieren que este uso del aoristo es propio de máximas y sentencias, de comparaciones épicas, de la descripción de costumbres y de la formulación de verdades de carácter general. Tal vez debería añadirse a esta nómina las construcciones de movimiento virtual.

\section{Futuro general o "gnómico»}

En los tratados científicos que describen principios de geometría abundan los futuros «gnómicos» ${ }^{38}$, que alternan con otras formas de referencia genérica en la formulación de axiomas:

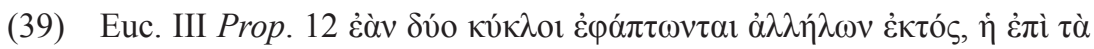

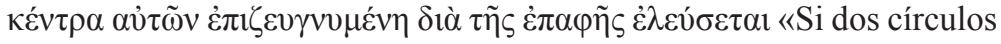
se tocan externamente, la recta ligada a ambos centros pasará por el punto de contacto».

Estas frases hacen referencia a un futuro meramente abstracto e hipotético, frecuentemente en periodos condicionales que refieren un axioma atemporal cuyo contenido es actualizable en cualquier momento. Kühner y Gerth 1898, p. 172, Anm. 1, citan el uso de un futuro de valor semejante en la descripción de costumbres por parte de Heródoto:

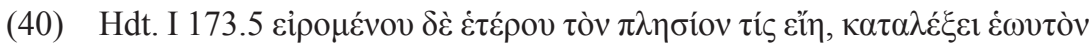

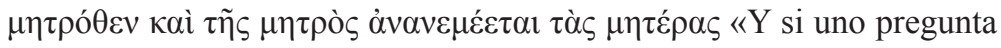
a un vecino quién es, se identificará a sí mismo a partir de su madre y nombrará las madres de su madre».

También se utiliza un futuro de segunda persona impersonal en descripciones geográficas con el formato de instrucciones a un viajero virtual, uso

\footnotetext{
${ }^{38}$ Kühner y Gerth 1898, p. 171; Stahl 1907, p. 141; Smyth 1920, §1914; Crespo, Conti y Maquieira 2003, p. 260. 
descrito por Méndez Dosuna 2009, p. 14. Stahl 1907, p. 141, explica el uso del futuro en la idea de que es previsible que los hechos se repitan a partir de la experiencia del hablante.

\section{Subjuntivo eventual}

Otro rasgo característico de los textos descriptivos es el empleo de subjuntivos eventuales en subordinadas relativas, condicionales y temporales ${ }^{39}$. En el periodo condicional de (39) el subjuntivo tiene referencia genérica. Además, hemos hallado bastantes casos de subjuntivo en temporales introducidas por $\dot{\varepsilon} \pi \varepsilon 1 \delta \alpha ́ v$, $\check{\sigma} \sigma \tau \varepsilon$ y $\check{\varepsilon} \omega \varsigma$. En las oraciones temporales se emplea subjuntivo con partícula tras una forma primaria cuando tiene sentido habitual, genérico o de verdad con carácter general (Goodwin 1889, p. 236). En (40) es posible una interpretación genérica:

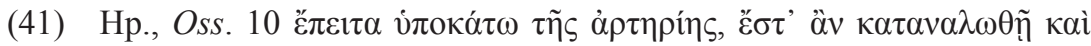

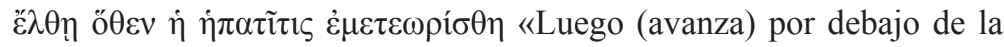
arteria hasta que desaparece y llega a donde la vena hepática se eleva».

Pero en (42-43) la expresión no es genérica, porque no hace referencia a la disposición de todos los miembros de una categoría hacia una actuación determinada. Se describe una entidad concreta y definida:

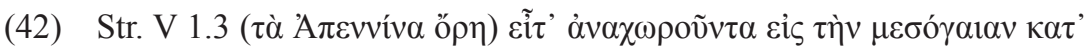

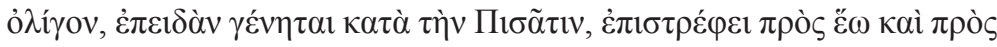

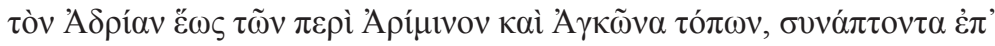

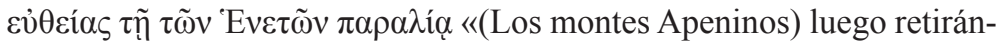
dose hasta casi la parte central, cuando se hallan frente a la Pisátide, se vuelven hacia oriente y hacia el Adriático hasta los lugares que rodean Arímino y Ancona, encontrándose en línea recta con la costa de los vénetos».

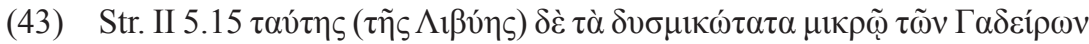

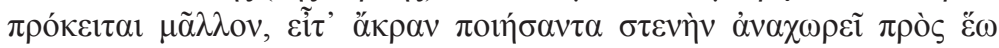

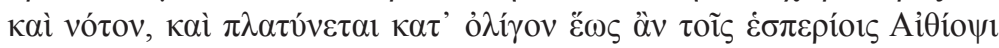

39 Kühner y Gerth 1898, p. 160; Stahl 1907, p. 131 ss.; Smyth 1920, §2409; Crespo, Conti y Maquieira 2003, p. 289 ss.; Wakker 1994, pp. 218-219. 
бuvółฺ̣ «Y la parte más occidental (de Libia) está ligeramente más a occidente que Cádiz, luego formando un cabo estrecho se retira hacia oriente y el sur y se ensancha ligeramente hasta que se encuentra con los etíopes occidentales»».

Tampoco son habituales en sentido estricto, porque no indican la disposición a actuar de una forma determinada según se deduce de un comportamiento sistemático previo. Sin duda apunta en la dirección correcta Méndez Dosuna 2009 , p. 20, cuando explica que «se trata de presentes habituales, que indican que el Trayecto en cuestión está siempre en disposición de ser recorrido». Aunque, como hemos comprobado, el sentido habitual no explica todos los usos que hemos tratado, ciertamente es el sentido más próximo al de estas estructuras que tratamos ahora. Es natural que los griegos hayan recurrido a la estructura propia de la descripción de costumbres para describir las propiedades permanentes de un objeto evocando metafóricamente una actividad meramente abstracta. Pensar que el movimiento se evoca como un hábito metafórico acaso sea ir demasiado lejos. Si autores como Goodwin hubieran tenido en cuenta ejemplos como éstos en la redacción de sus gramáticas, tal vez habrían incluido junto al matiz habitual y genérico el sentido virtual de estas expresiones. En todo caso el empleo de estos subjuntivos es, como los anteriores, un uso propio de la formulación de verdades de carácter general y este valor engloba tanto los usos genéricos, como los habituales y los estativos de base virtual.

\section{Conclusión}

A modo de conclusión ofrecemos una reformulación de la tesis central y aportación original de este trabajo. El sentido de los verbos de movimiento virtual implica un proceso metafórico que consiste en la proyección de un movimiento ficticio sobre una entidad inherentemente estática, proyección que permite la concepción de la entidad inerte como un ser que se desplaza. El carácter metafórico, junto a la función descriptiva o explicativa de esa metáfora, enmarca y explica al menos gran parte de las peculiaridades sintácticas y semánticas de los verbos de movimiento virtual y de sus restricciones y valores concretos en el plano de las categorías de voz, aspecto, tiempo y modo. Y lo hemos tratado de demostrar utilizando ejemplos y citando usos que no están descritos ni en las gramáticas al uso ni en estudios precedentes sobre el movimiento virtual. 


\section{BIBLIOGRAFÍA}

Adrados, F. R. 1992: Nueva sintaxis del griego antiguo, Madrid.

Allan, R. J. 2003: The middle voice in Ancient Greek: a study in polysemy, Ámsterdam.

Boel, G. de 1988: Goal accusative and object accusative in Homer, Bruselas.

Chantraine, P. 1963: Grammaire homérique, París.

Coulson, S. y Oakley, T. 2005: «Blended and coded meaning: literal and figurative meaning in cognitive semantics», Journal of Pragmatics 37, pp. 1510-1536.

Crespo, E., Conti, L. y Maquieira, H. 2003: Sintaxis del griego clásico, Madrid.

Fauconnier, G. 1997: Mappings in thought and language, Cambridge.

Goodwin, W. W. 1889: Syntax of the moods and tenses of the Greek verb, Londres.

Horrocks, G. C. 1983: Space and time in Homer: prepositional and adverbial particles in the Greek epic, Nueva York.

Iwata, S. 1996: «Motion and extent: two sides of the same coin», Studia Linguistica 50.3, pp. 256-282.

Jackendoff, R. 1983: Semantics and cognition, Cambridge.

Jiménez Delgado, J. M. 2008a: «Construcciones en voz activa en lugar de mediopasiva en Heródoto», Habis 39, pp. 7-30.

Jiménez Delgado, J. M. 2008b: «Metáfora y cambios de estructura semántica en el verbo griego antiguo», en Martínez Vázquez, R. y Jiménez Delgado, J. M. (eds.), Metáfora conceptual y verbo griego antiguo, Zaragoza, pp. 116-179.

Kroon, C. 2007: «Discourse Modes and the Use of Tenses in Ovid's Metamorphoses», en Allan, R. J. y Buijs, M. (eds.), The language of literature. Linguistic approaches to classical texts, Leiden, pp. 65-92.

Kühner, R. y Gerth, B. 1898: Ausführliche Grammatik der griechischen Sprache II, Hannover.

Langacker, R. W. 1986: «Abstract motion», Proceedings of the Annual Meeting of the Berkely Linguistics Society 12, pp. 455-471.

Langacker, R. W. 1990: «Subjectification», Cognitive Linguistics 1.1, pp. 5-38.

Langacker, R. W. 1991: Foundations of cognitive grammar, Vol. II: Descriptive application, Standford.

Langacker, R. W. 1999: «Virtual Reality», Studies in the Linguistic Sciences 29, pp. 77-103.

Luraghi, S. 2003: On the meaning of prepositions and cases. The expression of semantic roles in Ancient Greek, Ámsterdam-Filadelfia.

Martínez Vázquez, R. 1998: «The Ancient Greek concept of transitivity in a current cognitive semantic theory», en Martínez Vázquez, M. (ed.), Transitivity revisited, Huelva, pp. 15-35.

Martínez Vázquez, R. 2008a: «La base conceptual de la metáfora», en Martínez Vázquez, R. y Jiménez Delgado, J. M. (eds.), Metáfora conceptual y verbo griego antiguo, Zaragoza, pp. 11-57. 
Martínez Vázquez, R. 2008b: «Metáfora conceptual y marco predicativo: $\varphi \varepsilon ́ \rho \omega »$, en Martínez Vázquez, R. y Jiménez Delgado, J. M. (eds.), Metáfora conceptual y verbo griego antiguo, Zaragoza, pp. 58-115.

Martínez Vázquez, R. 2009: «Aspectos cognitivos del aspecto verbal: metonimia y presente por perfecto en griego antiguo», Habis 40, pp. 7-16.

Martínez Vázquez, R. 2011: «Tipología textual, adverbios conjuntivos y la Historia de Tucídides», en Carande Herrero, R. y López-Cañete Quiles, D. (eds.), Pro Tantis Redditur. Homenaje a Juan Gil en Sevilla, Sevilla, pp. 95-114.

Matlock, T. 2004: «The conceptual motivation of fictive motion», en Radden, G. y Dirven, R. (eds.), Studies in linguistic motivation, Berlín, pp. 221-248.

Matsumoto, Y. 1996: «Subjective motion in English and Japanese verbs», Cognitive Linguistics 7.2, pp. 183-226.

Méndez Dosuna, J. 2009: «Movimiento ficticio en griego antiguo: tras las huellas del viajero (in)visible», RSEL 39.1, pp. 5-32.

Rodenbusch, E. 1911: «Präsentia in perfektischer Bedeutung», IF 28, pp. 252-285.

Rojo, A. y Valenzuela, J. 2003: «Fictive motion in English and Spanish», International Journal of English Studies 3.2, pp. 125-151.

Schwyzer, E. y Debrunner, A. 1950: Griechische Grammatik II, Múnich.

Sivonen, J. 2005: «An exercise in cognitive lexical semantics: the case of the Finnish motion verb kiertää», SKY Journal of Linguistics 18, pp. 311-340.

Smyth, H. W. 1920: A Greek grammar for colleges, Cambridge.

Stahl, J. M. 1907: Kritisch-historische Syntax des griechischen Verbums der klassischen Zeit, Hildesheim.

Takahashi, K. 2001: «Access path expressions in Thai», en Cienki, A., Luke, B. J. y Smith, M. B. (eds.), Conceptual and Discourse Factors in Linguistic Structure, Stanford, pp. 237-252.

Talmy, L. 1983: «How language structures space», en Pick, H. L. Jr. y Acredolo, L. P. (eds.), Spatial orientation: theory, research, and application, Nueva York, pp. 225-282.

Talmy, L. 1996: "Fictive motion in language and "ception"», en Bloom, P., Peterson, M. A., Nadel, L. y Garrett, M. F. (eds.), Language and space, Cambridge, pp. 211-276.

Talmy, L. 2000: Toward a cognitive semantics I, Boston.

Wakker, G. 1994: Conditions and conditionals. An investigation of Ancient Greek, Ámsterdam.

Fecha de recepción de la primera versión del artículo: 28/06/2010

Fecha de aceptación: 01/12/2010

Fecha de recepción de la versión definitiva: 20/01/2011 